\title{
Quantifying variation in female internal genitalia: no evidence for plasticity in response to sexual conflict risk in a seed beetle
}

Blake W. Wyber ${ }^{1}$, Liam R. Dougherty ${ }^{1,2}$, Kathryn McNamara ${ }^{1,3}$, Andrew Mehnert ${ }^{4,5}$, Jeremy Shaw ${ }^{4,5}$, Joseph L. Tomkins ${ }^{1}$ \& Leigh W. Simmons ${ }^{1}$

${ }^{1}$ Centre for Evolutionary Biology, School of Biological Sciences, The University of Western Australia, 35 Stirling Highway, Crawley WA 6009, Australia; ${ }^{2}$ Institute of Infection, Veterinary and Ecological Sciences, University of Liverpool, Liverpool, L69 7RB, UK;

${ }^{3}$ School of BioSciences, The University of Melbourne, Parkville, Victoria, 3010; ${ }^{4}$ Centre for Microscopy, Characterisation and Analysis , The University of Western Australia, Perth WA 6009, Australia; ${ }^{5}$ National Imaging Facility, Australia 


\section{Abstract}

2 Sexually antagonistic coevolution can drive the evolution of male traits that harm females,

3 and female resistance to those traits. While males have been found to vary their harmfulness

4 to females in response to social cues, plasticity in female resistance traits remains to be

5 examined. Here we ask whether female seed beetles Callosobruchus maculatus are capable

6 of adjusting their resistance to male harm in response to the social environment. Among seed

7 beetles, male genital spines harm females during copulation and females might resist male

8 harm via thickening of the reproductive tract walls. We develop a novel Micro-CT imaging

9 technique to quantify female reproductive tract thickness in 3-dimensional space, and compared the reproductive tracts of females from populations that had evolved under high and low levels of sexual conflict, and for females reared under a social environment that predicted either high or low levels of sexual conflict. We find little evidence to suggest that females can adjust the thickness of their reproductive tracts in response to the social environment. Neither did evolutionary history affect reproductive tract thickness. Nevertheless, our novel methodology was capable of quantifying fine-scale differences in the internal reproductive tracts of individual females, and will allow future investigations into the

17 internal organs of insects and other animals.

Key Words: female genital evolution, sexual conflict, sexually antagonistic coevolution, phenotypic plasticity, experimental evolution, sex ratio 
21 


\section{Introduction}

Evolutionary conflict between the sexes can arise over the expression of traits that improve the fitness of one sex but are detrimental to the fitness of their sexual partners (Arnqvist and Rowe 2005; Parker 2006). Sexual selection favours traits in males that confer greater fertilisation success regardless of the outcomes for females, while antagonistic selection on females can favour morphological and behavioural traits that function to resist harm induced by males (Stockley 1997; Edvardsson and Tregenza 2005). Harmful traits in males are diverse in form and include: male harassment during courtship and mating (den Hollander and Gwynne 2009; Rankin et al. 2011), infanticide (Arnqvist and Rowe 2005; Parker 2006), and toxic ejaculates (Chapman et al. 1995; den Hollander and Gwynne 2009). One conspicuous form of sexual conflict is the damage imposed to the female's reproductive tract by male genitalia during copulation (Lange et al. 2013). Genital damage during copulation is found across a variety of taxa, particularly within arthropod lineages (Crudgington and SivaJothy 2000; Blanckenhorn et al. 2002; Kamimura 2010; Tatarnic and Cassis 2010; Kamimura 2012; Tatarnic et al. 2014) and is considered to be a by-product of male traits enhancing fertilisation success, rather than harm directly benefitting males (Morrow et al. 2003; Hotzy and Arnqvist 2009; Grieshop and Polak 2014; McNamara et al. 2020).

The evolution of harmful traits in one sex is expected to generate selection on the opposite sex favouring the evolution of traits to resist harm, initiating sexually antagonistic coevolution between the sexes (Arnqvist and Rowe 1995; Rice 1996; Arnqvist and Rowe 2002a; Tatarnic and Cassis 2010; Dougherty et al. 2017). While comparative evidence suggests that coevolution of harmful male traits and female resistance traits is widespread (Brennan et al. 2007; Perry and Rowe 2012; Hopwood et al. 2016; Dougherty et al. 2017), whether these traits can respond plastically to an individual's environment has received less attention. Males of a wide range of taxa have been found to adjust ejaculate size, composition 
and quality in response to exposure to rival males (Gage and Baker 1991; Pilastro et al. 2002;

Evans et al. 2003; Simmons et al. 2007; Thomas and Simmons 2007; DelBarco-Trillo 2011), and recent work suggests that male genital morphology can be adjusted in response to the competitive environment experienced during sexual development (Brennan et al. 2017; André et al. 2018). Thus, males exhibit phenotypic plasticity in sexual traits that can be costly for females. Firman and Simmons (Firman and Simmons 2013) found that female house mice exposed to greater levels of sperm competition risk produced ova with lower fertilizability, presumably to mitigate against the threat of polyspermy when males compete for fertilizations. This finding suggests that females too may be capable of responding to the socio-sexual environment, and reduce the costs associated with male competition. Using a model species in which sexual conflict has been widely documented, we test the hypothesis that sexual conflict favours the evolution of phenotypic plasticity in female resistance traits in response to the immediate risk of sexual conflict.

Seed beetles (Callosobruchus maculatus) are a widely used model species for sexual conflict studies (Zuk et al. 2014). Research has focused on the role and outcomes of male harmfulness on the persistence of polyandry, the effect of sperm competition on male ejaculate investment, the effects of kinship on male harmfulness, and a wide array of other topics (Rönn et al. 2006; Hotzy and Arnqvist 2009; Wilson and Tomkins 2015; Lymbery and Simmons 2017; McNamara et al. 2020). The aedeagus (intromittent organ) is covered in sclerotized spines that perforate the female reproductive tract during copulation, inflicting significant scarring (Crudgington and Siva-Jothy 2000; Dougherty and Simmons 2017). The degree of reproductive tract damage negatively impacts both female longevity and female reproductive success (den Hollander and Gwynne 2009) but promotes male fertilisation success (Hotzy and Arnqvist 2009). Reproductive tract damage facilitates the transport of accessory seminal compounds into the female bloodstream, which improve male competitive 
fertilisation success (Hotzy et al. 2012; Rönn and Hotzy 2012; Yamane et al. 2015).

Therefore, the harm to females in this species seems to be a side-effect of selection for competitive male fertilization success (Hotzy et al. 2012; McNamara et al. 2020). The thickness of the female reproductive tract wall appears to have evolved under sexually antagonistic coevolution to resist the harmful effects of male genital spines. Thus, across populations (Dougherty et al. 2017) and species (Ronn et al. 2007), female reproductive tract volume has been found to be positively correlated with male penile spine length, providing one of the few empirical examples of sexually antagonistic coevolution (Dougherty et al. 2017). Moreover, male C. maculatus appear to adjust their copulatory behaviour (Wilson et al. 2014; Wilson and Tomkins 2014) and amount of harm imposed on females in response to their social environment [37,46, but see 47].

Here, we investigate whether female C. maculatus exposed to greater risk of sexual conflict can respond by adjusting the thickness of the reproductive tract to minimise anticipated male harm. We used a manipulation of the social environment to vary the immediate risk of sexual conflict: we had two treatments within which we manipulated both larval density, and the adult sex-ratio to simulate either a high or low sexual conflict risk. Additionally, we employed an experimental evolution design to test whether the thickness of the female reproductive tract diverged between populations of beetles evolving under a maleor female-biased sex-ratio. Assessment of reproductive tract morphology in this species has previously focused on either the thickness of the tract estimated from a small number of histological sections (Ronn et al. 2007), or the total volume of tissue across the entire tract via 3-dimensional analysis of Micro-CT images (Dougherty et al. 2017). Although tissue volume may capture large-scale changes in female investment, it does not capture fine-scale changes in tract morphology. Here, we developed a novel technique to measure variation in the thickness of the female reproductive tract at different locations along its length using Micro- 
97 CT data. We predicted that: 1) females in populations evolving under a male-biased sex-ratio will have thicker reproductive tract walls than those evolving under a female-biased sex ratio as a result of sexually antagonistic coevolution; and 2) females exposed to high-density larval environments and a male-biased social environment during development will develop thicker reproductive tract walls compared to females from low density larval environments exposed to a female-biased social environment, in anticipation of an increased risk of sexual conflict.

\section{Methods}

(a) Study population

The stock population of $C$. maculatus used for this study was derived originally from a population held by the CSIRO in 2005, which was itself founded by individuals found as agricultural pests. Both stock and experimental populations were maintained at $30^{\circ} \mathrm{C}$ under a 12:12 hour day/night regime for the duration of the experiment (McNamara et al. 2016). For further information regarding the stock population see (Wilson et al. 2014).

\section{(b) Experimental evolution}

Experimental evolution lines were produced and maintained as described in McNamara et al. [18]. In brief, individuals from the stock population were used to create six experimental evolution lines. These lines were randomly assigned to one of two treatments, with either a male- or female-biased sex ratio. Each generation consisted of 120 individuals, with an 80:40 male to female ratio for male-biased lines, and vice versa for female-biased lines. To control for potential differences in larval competition between treatments, female-biased populations received 200g of mung beans (Vigna radiata) for oviposition, while male-biased populations 
received 100g. Each new generation was created by isolating 300 beans per population within ventilated 1.5mL Eppendorf tubes. After the required adults had emerged, sex-biased populations were again formed. This procedure was continued for 47 generations, after which populations were placed under common garden conditions for two generations with sex-ratio parity to remove the potential for any non-genetic parental effects. Following the second generation of common-garden breeding, beans were placed within ventilated Eppendorf tubes.

\section{(c) Social manipulation}

Isolated beans were assigned to one of two social manipulations designed to alter an individual's perception of future sexual conflict, either high-risk or low-risk. Evidence suggests that seed beetle larvae are able to determine population density before emergence from their beans via vibrations (Utida 1972; Thanthianga and Mitchell 1987). For this experiment we elected to use mung beans (Vigna radiata) as the larval host species to increase surface area compared to their larger, traditional host species (Vigna unguiculata), and thereby improve the transmission of vibrational cues to focal individuals. Therefore, in the high-risk treatment, five infested beans, each containing two larvae (infestation density can be assessed as eggs remain visible on the surface of the bean), were placed within an Eppendorf tube (resulting in ten larvae maximally per tube). Eppendorf tubes were checked daily for beetle emergence. Females that emerged synchronously with a male were discarded from the experiment to ensure focal females had standardised pre-mating social exposure and remained unmated. Females that emerged alone were placed within the lid of a $1.5 \mathrm{~mL}$

Eppendorf tube, separated from four stock males and two stock females within the body of the tube via cotton mesh. The mesh allowed focal females to detect the presence of 
individuals but prevented them from copulating with the males. Previous studies have shown that this methodology elicits phenotypic responses to the social environment in both sexes of C. maculatus (van Lieshout et al. 2014; Wilson and Tomkins 2014). Thus, high-risk females experienced high larval density and a male-biased sex ratio. For the low-risk treatment, a single infested bean, containing a single larva, was placed within an Eppendorf tube containing four un-infested beans. Following emergence, females were placed within an Eppendorf tube separated from two stock males and four stock females. Thus, low-risk females experienced a low larval density and a female-biased sex ratio prior to mating. In the low-risk social treatment, we aimed to provide cues that were representative of naturalistic conditions. Given the high densities experienced by C. maculatus when infesting food stores, we therefore exposed females to a small number of males rather than no males. For both treatments, females were removed after 24 hours and then allowed to mate once with a stock male. Females were isolated for a further 24 hours post-copulation, and then euthanized by freezing. Although we might expect adjustments in the reproductive tract to most likely occur Wilson and Tomkins 2014).

(d) Micro CT-scanning and tomographic reconstruction

A total of five females per population-by-social-treatment combination were selected for Micro-CT scanning. Therefore, a total of 60 individuals were scanned for the purposes of this 
et al. [22], with the exception that we used formalin for tissue fixation rather than paraformaldehyde (see detailed pre-scan methodology in the online supplementary material).

Samples were scanned using a ZEISS Xradia Versa 520 X-ray microscope housed at the University of Western Australia Centre for Microscopy, Characterisation and Analysis. Samples were suspended in $100 \%$ ethanol and mounted in heat-sealed pipette tips with waxcovered tops in groups of 3-5 for scanning. Abdomens were arranged vertically and scanned sequentially from the highest abdomen to the lowest. Source voltage and power of initial scans $(\mathrm{n}=11)$ was set at $40 \mathrm{kV}$ and $3 \mathrm{~W}$. However, source stability was compromised for an extended period of time, so the remaining scans were conducted at $60 \mathrm{kV}$ and $5 \mathrm{~W}(\mathrm{n}=43)$ to ensure that scan quality remained stable (details of the scanning procedures can be found in the online supporting material). A total of six samples could not be analysed due to low scan quality, leaving a sample size of 54 for image analysis.

\section{(e) Image analysis}

Images were analysed blind with regard to both the population of origin and social treatment. The analyses were performed using a combination of three custom-written FIJI (Schindelin et al. 2012; Schneider et al. 2012; Legland et al. 2016) scripts and Amira 6.2 (Thermo-Fisher Scientific, U.S.A.). We first selected a consistent region of interest within each reproductive tract, which was marked by the entrance of the spermathecal duct into the reproductive tract at one end (Figure 1a) and the first occurrence of bursal teeth on the other (Figure 1b) This region was chosen because it sustains the greatest damage during copulation (Dougherty et al. 2017). Within this region of interest, we computed the 3D thickness of the dorsal and ventral reproductive tract walls based on the local thickness definition proposed by Dougherty and Kunzelmann (Dougherty and Kunzelmann 2007) (see online Supplementary material for 
detailed methodology). According to this definition the thickness at any point within an object, in our case the tract walls, is the diameter of the largest sphere that fits inside the object and at the same time contains the point (Figure 1c). Further, we differentiated investment in the upper and lower reproductive tract by placing a horizontal plane running through the lumen's centroid, which allowed us to reliably define the upper and lower regions of tracts among all sampled individuals (Figure 1d).

\section{(f) Statistical Analyses}

All statistical analyses were conducted in R (v 3.5.3) (R Development Core Team 2016). Normality of data was confirmed with Shapiro-Wilk’s tests. Further, Bartlett's test for homogeneity of variance was found to be non-significant in all cases.

Pearson’s correlation tests using the 'ppcor’ R package (Kim 2015) found significant correlations between the mean, minimum and maximum thickness values from the upper and lower reproductive tract. Repeatability analyses were conducted for all measurements by extracting measures of 9 individuals on each of three separate occasions and analysing the data using the R package 'rptR' (Stoffel et al. 2017). We found significant repeatability (R > 0.6) in mean and maximum tract thickness but not in minimum tract thickness (see Table S2 in the online Supplementary Material). We therefore conducted principal components analysis using the 'FactoMineR' R package, excluding upper and lower minimum thickness (Lê et al. 2008 Mar 18). One principal component (PC1) had an eigenvalue greater than 1 and was extracted for further analysis. PC1 was used as the dependent variable using a Gaussian linear mixed model that included female weight as a covariate. Following Arnqvist (Arnqvist 2020), replicate population was included as a random factor within which an interaction between female weight and social treatment was fitted as a random slope. The random slope 
212 allows for the interaction to vary across the differing populations (Arnqvist 2020). The source

213 voltage was also included in this model as a random factor to control for any variance in trait

214 estimates that might have arisen from the use of different voltage settings during scanning.

215 The significance of our treatments, random factors and interaction effects were tested using

216 Kenward-Roger F-tests using the R package 'pbkrtest'(Halekoh and Højsgaard 2014). Nonsignificant interactions that did not improve model fit were subsequently removed from final models. Effect sizes were estimated as the standardised Pearson’s correlation coefficients (Table 3) using the 'effectsize’ package in R. Estimated marginal means for each level of the two treatments were produced via the package 'emmeans'. The data were explored for outliers using robust kernel-based outlier factor algorithms within the 'OutlierDetection’ R package $(k=3$, bootstraps $=50,000)$. No outliers were identified .

\section{Results}

224 The first principal component explained $72 \%$ of the variance in reproductive tract thickness (Table 1) and was loaded equally by the mean and maximum thickness from both the upper and lower reproductive tract. There was no significant impact of evolutionary history or

227 social treatment on female tract (Table 2). All interaction combinations between evolutionary history, social treatment and weight were found to be non-significant and were dropped from the final model.

\section{Discussion}


We developed a novel Micro-CT imaging method to measure the thickness of the reproductive tract walls of female $C$. maculatus seed beetles, from populations that had evolved under a male- or female-biased population sex ratio for 47 generations, and which were subsequently exposed to a social environment which conveyed either a high or low-risk of sexual conflict. Previous studies of this species have found that males can adjust their harmfulness to females in response to their social environment [43-45, but see: 56]. There was no effect of evolutionary history on the overall thickness of the female reproductive tract, nor was there any effect of the social environment. Our data therefore suggests that female reproductive tracts may not respond to variation in the risk of sexual conflict.

Previous studies using C. maculatus have similarly failed to find evolutionary responses in females to experimental manipulations of sexual conflict. Gay et al. [57] showed that after 90 generations of enforced monogamy, the re-introduction of polyandry over 30 generations resulted in the evolution of harmful males but not resistant females. Similarly, after 8 generations of enforced monogamy it was found that the elaboration of male penile spines decreased, as might be predicted for a costly trait that functions in the context of competitive reproductive success. However, the morphology of the teeth found within the female genital tract failed to exhibit a correlated response to enforced monogamy (Cayetano et al. 2011). Our results are also consistent with recent findings of McNamara et al. [18] who utilised the same experimental evolution lines used in the current study. McNamara et al. (McNamara et al. 2020) found that although males evolving under a male-biased sex ratio evolved to be more harmful, females from populations evolving under a male-biased sex ratio experienced comparable reproductive tract scarring to females from populations evolving under a female-biased sex ratio. We found no evidence that sexual conflict intensity results in an evolutionary divergence in female reproductive tract thickness. Collectively, these findings suggest that either females fail to coevolve as readily as males, or that female 
reproductive tract coevolution is more difficult to detect (Gay et al. 2010; Cayetano et al. 2011; McNamara et al. 2020).

The findings of these experimental evolution studies are in contrast to comparative studies that have found evidence for sexually antagonistic coevolution between male harmfulness and female resistance to harm among C. maculatus populations and among Callosobruchus species more widely (Ronn et al. 2007; Dougherty et al. 2017). Further, the coevolution of resistance traits and male persistence has been identified in comparative studies of other arthropod taxa such as water striders (Heteroptera: Gerridae) (Arnqvist and Rowe 2002b) and diving beetles (Coleoptera: Dytiscidae) (Bergsten et al. 2008). The apparent inability of female seed beetle reproductive tracts to respond evolutionarily to relatively short-term manipulations in sexual conflict might be attributable to the difficulty in detecting sexually antagonistic coevolution at a particular point in time (Arnqvist and Rowe 2005; Perry and Rowe 2012; Kokko and Jennions 2014). This suggestion could explain why females seemingly did not respond to our selection treatments, as it is clear that divergence in male harmfulness was present in these populations 15 generations earlier (McNamara et al. 2020). We are unable to say whether females from populations experiencing differing levels of sexual conflict might have developed alternative methods of resistance, such as higher immunocompetence (Hangartner et al. 2015). Previous studies utilising the same experimental evolution lines have shown that individuals derived from the male-biased lines exhibit reduced immune function (van Lieshout et al. 2014). These results are indicative of a resource trade-off between immune function and reproductive investment fuelled by the costs of high intensity sexual conflict. Although the method by which Gay et al. [57] and McNamara et al. (McNamara et al. 2020) altered sexual conflict intensity differed, both studies found that females from populations that experienced higher conflict were better able to counter-act the negative impact of mating on their fitness. However, it is clear that the 
method by which female $C$. maculatus accomplish this is not via genital morphology, female kicking behaviour, or through improved immunity (van Lieshout et al. 2014; McNamara et al. 2020). Finally, it may be that inbreeding depression was responsible for impeding divergence among our lines. This seems unlikely however, because a hallmark of inbreeding is reduced fitness, which would have been observed as reduced fecundity for females from the femalebiased lines. A previous study using the same experimental evolution lines (McNamara et al. 2020) showed no evidence that females from female-biased lines experienced reduce fitness compared to those from male-biased lines.

We found that the social environment had no impact on female reproductive tract thickness. This is the only study to have investigated whether females are able to plastically respond to sexual conflict risk by altering reproductive tissue dimensions. There are several reasons why females might have failed to respond to our manipulation of their social environment. First, it is possible that females were unable to adjust the reproductive tract in response to our environmental cues. Females were given a proxy for sexual conflict risk via manipulations of larval density during development, but this cue may have been insufficient to provoke a plastic response. Second, the larval and pupal stages are critical to the development of insect reproductive organs (Happ 1992). This is demonstrated by the longlasting impacts of larval food availability on adult reproductive output in both female and male insects (Bauerfeind and Fischer 2005; Engels and Sauer 2007). Females were exposed to direct signals of increased sexual conflict risk (via sex-ratio) in their adult stage, postpupation, but they may be unable to make plastic adjustments after adult emergence. Third, it is also possible that our measures of tract thickness do not capture important qualitative variation. For example, it may be that females can also plastically adjust the elasticity of the reproductive tract through the incorporation of resilin, a compound shown to improve tolerance to cuticle perforation in bed bugs (Cimex lectularius)(Michels et al. 2015). Finally, 
it may be that females plastically responded to our social manipulation, but due to the logistical limitations placed on us with respect to the numbers of females we could scan, we were unable to capture any differences among them. However, given that our repeatability analysis revealed significant variation among females in reproductive tract thickness, we can be certain that we are able to detect variation in tract thickness using this novel technique. If there were an effect of the social environment on the reproductive tract thickness, the effect size estimated in our analysis suggest that it is small and would require a large sample size in order to detect significance.

Although our measure of female resistance traits is limited to a quantitative measure of reproductive tract thickness, it nonetheless offers a significant advance over previous studies. Previous studies utilising 3-dimensional analysis of Micro-CT scans have measured total reproductive tract volume, which is effective at controlling for tract shape and size effects, but cannot identify fine-scale changes in morphology within the reproductive tract (Dougherty et al. 2017). Our current method overcomes this challenge by allowing us to identify variation in thickness in the upper and lower regions of the reproductive tract in 3dimensional space. Overall, our novel method for measuring reproductive tract thickness shows promise in its ability to detect fine-scale differences in internal structures of the female reproductive tract, and promises to be a valuable tool in the long-awaited study of female genital morphology across numerous species.

In conclusion, we provide no evidence for plastic adjustments of reproductive tract thickness, a trait known to have coevolved with male-imposed genital damage among populations and species of Callosobruchus. Moreover, we found that populations that had evolved under intense sexual conflict failed to diverge in female genital morphology. The coevolution of male and female genital traits among populations suggests ample genetic variation in tract morphology may exist, however among-population genetic variance does 
not necessarily reflect within-population variation (Hoffmann et al. 2003). Therefore, the lack of divergence in tract morphology across our evolution lines may reflect a lack of withinpopulation genetic variation for this trait. Studies of sexual selection acting on female genitalia typically lag behind those focussed on male traits (Kokko and Jennions 2014; Sloan and Simmons 2019). Investigating female traits and their responses to male harmfulness can broaden our understanding of sexual selection and sexual conflict. Further research needs to focus on the female perspective if we are to quantify the pervasiveness and intensity of female responses to sexual conflict. The barriers to such research are slowly dissipating with the advent of innovative new technologies, such as Micro-CT scanning, that allow more effective measurement of female traits. As technologies become more accessible and cheaper to employ, increased sample sizes will be possible so that future studies have the power to detect variation in these minute structures. We believe that the present study provides a viable methodological approach for further investigations into plastic adjustments in female reproductive tracts, which is flexible enough to identify small-scale variation.

\section{Funding}

B. W. W. is supported by the Research Training Program and the School of Biological Sciences, UWA. L.R.D was funded by the Australian Research Council to Leigh Simmons (DP-130100618). K.B.M was supported by the Australian Research Council (DE160100097).

\section{Acknowledgements}

Jessica Hadlow provided assistance with statistical analysis. The authors acknowledge the facilities, scientific and technical assistance of Microscopy Australia (MA), and the scientific and technical assistance of the National Imaging Facility (NIF), both National Collaborative 
Research Infrastructure Strategy (NCRIS) capabilities, at the Centre for Microscopy, Characterisation and Analysis (CMCA), The University of Western Australia.

\section{References}

André GI, Firman RC, Simmons LW. 2018. Phenotypic plasticity in genitalia: baculum shape responds to sperm competition risk in house mice. Proc R Soc B Biol Sci. 285(1882):20181086. doi:10.1098/rspb.2018.1086.

Arnqvist G. 2020. Mixed Models Offer No Freedom from Degrees of Freedom. Trends Ecol Evol. 35(4):329-335. doi:https://doi.org/10.1016/j.tree.2019.12.004.

Arnqvist G, Rowe L. 1995. Sexual conflict and arms races between the sexes: a morphological adaptation for control of mating in a female insect. Proc R Soc London Ser B Biol Sci. 261(1360):123-127. doi:10.1098/rspb.1995.0126.

Arnqvist G, Rowe L. 2002a. Antagonistic coevolution between the sexes in a group of insects. Nature. 415(February):787-789.

Arnqvist G, Rowe L. 2002b. Correlated evolution of male and female morphologies in water striders. Evolution (N Y). 56(5):936-947. doi:10.1111/j.0014-3820.2002.tb01406.x.

Arnqvist G, Rowe L. 2005. Sexual Conflict. Rowe Locke, editor. Princeton, N.J.: Princeton University Press (Monographs in behavior and ecology).

Bauerfeind SS, Fischer K. 2005. Effects of food stress and density in different life stages on reproduction in a butterfly. Oikos. 111(3):514-524. doi:https://doi.org/10.1111/j.00301299.2005.13888.x.

Berg EC, Lind MI, Monahan S, Bricout S, Maklakov AA. 2019. Kin but less than kind: within-group male relatedness does not increase female fitness in seed beetles. Proc R Soc B Biol Sci. 286(1910):20191664. doi:10.1098/rspb.2019.1664. 
Bergsten J, Töyrä A, Nilsson AN. 2008. Intraspecific variation and intersexual correlation in secondary sexual characters of three diving beetles (Coleoptera: Dytiscidae). Biol J Linn Soc. 73(2):221-232. doi:10.1111/j.1095-8312.2001.tb01359.x.

Blanckenhorn WU, Hosken DJ, Martin OY, Reim C, Teuschl Y, Ward PI. 2002. The costs of copulating in the dung fly Sepsis cynipsea. Behav Ecol. 13(3):353-358. doi:10.1093/beheco/13.3.353.

Brennan PLR, Gereg I, Goodman M, Feng D, Prum RO. 2017. Evidence of phenotypic plasticity of penis morphology and delayed reproductive maturation in response to male competition in waterfowl. Auk. 134(4):882-893. doi:10.1642/AUK-17-114.1.

Brennan PLR, Prum RO, McCracken KG, Sorenson MD, Wilson RE, Birkhead TR. 2007. Coevolution of Male and Female Genital Morphology in Waterfowl. PLoS One. 2(5):e418.

Cayetano L, Maklakov AA, Brooks RC, Bonduriansky R. 2011. Evolution of male and female genitalia following release from sexual selection. Evolution (N Y). 65(8):2171-2183. doi:10.1111/j.1558-5646.2011.01309.x.

Chapman T, Liddle LF, Kalb JM, Wolfner MF, Partridge L. 1995. Cost of mating in Drosophila melanogaster females is mediated by male accessory gland products. Nature. 373(6511):241-244. doi:10.1038/373241a0.

Crudgington HS, Siva-Jothy MT. 2000. Genital damage, kicking and early death. Nature. 407(6806):855-856. doi:10.1038/35038154.

DelBarco-Trillo J. 2011. Adjustment of sperm allocation under high risk of sperm competition across taxa: a meta-analysis. J Evol Biol. 24(8):1706-1714. doi:10.1111/j.1420-9101.2011.02293.x.

Dougherty LR, van Lieshout E, McNamara KB, Moschilla JA, Arnqvist G, Simmons LW. 2017. Sexual conflict and correlated evolution between male persistence and female resistance traits in the seed beetle Callosobruchus maculatus. Proc R Soc B Biol Sci. 284(1855):20170132. doi:10.1098/rspb.2017.0132. 
harm and female kicking during traumatic mating in seed beetles. Proc R Soc B Biol Sci. 284(1856).

407 doi:10.1098/rspb.2017.0550.

408

Dougherty R, Kunzelmann K-H. 2007. Computing Local Thickness of 3D Structures with ImageJ. Microsc Microanal. 13(S02):1678-1679. doi:DOI: 10.1017/S1431927607074430.

Edvardsson M, Tregenza T. 2005. Why do male Callosobruchus maculatus harm their mates? Behav Ecol. 16(4):788-793. doi:10.1093/beheco/ari055.

Engels S, Sauer KP. 2007. Energy beyond the pupal stage: Larval nutrition and its long-time consequences for male mating performance in a scorpionfly. J Insect Physiol. 53(7):633-638. doi:https://doi.org/10.1016/j.jinsphys.2007.05.003.

Evans JP, Pierotti M, Pilastro A. 2003. Male mating behavior and ejaculate expenditure under sperm competition risk in the eastern mosquitofish. Behav Ecol. 14(2):268-273.

417 Firman RC, Simmons LW. 2013. Sperm competition risk generates phenotypic plasticity in ovum fertilizability. Proc R Soc B Biol Sci. 280(1772):20132097. doi:10.1098/rspb.2013.2097. Entomol. 16(3):331-337. doi:10.1111/j.1365-2311.1991.tb00224.x.

Gay L, Hosken DJ, Eady PE, Vasudev R, Tregenza T. 2010. The evolution of harm—effect of sexual conflicts and population size. Evolution (N Y). 65(3):725-737. doi:10.1111/j.15585646.2010.01181.x.

Grieshop K, Polak M. 2014. Evaluating the post-copulatory sexual selection hypothesis for genital evolution reveals evidence for pleiotropic harm exerted by the male genital spines of Drosophila ananassae. J Evol Biol. 27(12):2676-2686. doi:10.1111/jeb.12524. for Tests in Linear Mixed Models $\square$ The R Package pbkrtest. J Stat Software; Vol 1, Issue 9 . doi:10.18637/jss.v059.i09. 
Hangartner S, Michalczyk Ł, Gage MJG, Martin OY. 2015. Experimental removal of sexual selection leads to decreased investment in an immune component in female Tribolium castaneum. Infect Genet Evol. 33:212-218. doi:https://doi.org/10.1016/j.meegid.2015.05.005.

Happ GM. 1992. Maturation of the Male Reproductive System and its Endocrine Regulation. Annu Rev Entomol. 37(1):303-320. doi:10.1146/annurev.en.37.010192.001511. in a Rainforest Drosophila Species. Science (80- ). 301(5629):100 LP - 102. doi:10.1126/science.1084296.

den Hollander M, Gwynne DT. 2009. Female fitness consequences of male harassment and copulation in seed beetles, Callosobruchus maculatus. Anim Behav. 78(5):1061-1070. doi:10.1016/j.anbehav.2009.06.036.

Hopwood PE, Head ML, Jordan EJ, Carter MJ, Davey E, Moore AJ, Royle NJ. 2016. Selection on an antagonistic behavioral trait can drive rapid genital coevolution in the burying beetle, Nicrophorus vespilloides. Evolution. 70(6):1180-1188. doi:10.1111/evo.12938.

Hotzy C, Arnqvist G. 2009. Sperm Competition Favors Harmful Males in Seed Beetles. Curr Biol. 19(5):404-407. doi:10.1016/j.cub.2009.01.045.

Hotzy C, Polak M, Rönn JL, Arnqvist G. 2012. Phenotypic Engineering Unveils the Function of Genital Morphology. Curr Biol. 22(23):2258-2261. doi:https://doi.org/10.1016/j.cub.2012.10.009.

Kamimura Y. 2010. Copulation anatomy of Drosophila melanogaster (Diptera: Drosophilidae): doi:10.1007/s00435-010-0109-5.

Kamimura Y. 2012. Correlated evolutionary changes in Drosophila female genitalia reduce the possible infection risk caused by male copulatory wounding. Behav Ecol Sociobiol. 66(8):1107-1114. doi:10.1007/s00265-012-1361-0. 
456 Kokko H, Jennions MD. 2014. The relationship between sexual selection and sexual conflict. Cold

457 Spring Harb Perspect Biol. 6(9). doi:10.1101/cshperspect.a017517.

458 Lange R, Reinhardt K, Michiels NK, Anthes N. 2013. Functions, diversity, and evolution of traumatic 459 mating. Biol Rev. 88(3):585-601. doi:10.1111/brv.12018.

460 Lê S, Josse J, Husson F. 2008 Mar 18. FactoMineR: An R Package for Multivariate Analysis. J Stat 461 Software; Vol 1, Issue 1.

462 Legland D, Arganda-Carreras I, Andrey P. 2016. MorphoLibJ: integrated library and plugins for 463 mathematical morphology with ImageJ. Bioinformatics. 32(22):3532-3534.

464 doi:10.1093/bioinformatics/btw413. Immunocompetence Under Intense Sexual Selection. Evolution (N Y). 68(9):2550-2558. doi:10.1111/evo.12422.

Lymbery SJ, Simmons LW. 2017. Males harm females less when competing with familiar relatives. Proc R Soc B Biol Sci. 284(1867):20171984. doi:10.1098/rspb.2017.1984.

Lymbery SJ, Tomkins JL, Simmons LW. 2019. Male responses to sperm competition when rivals

471 vary in number and familiarity. Proc R Soc B Biol Sci. 286(1895):20182589.

472 doi:10.1098/rspb.2018.2589.

473 McNamara KB, Robinson SP, Rosa ME, Sloan NS, van Lieshout E, Simmons LW. 2016. Male-biased 474 sex ratio does not promote increased sperm competitiveness in the seed beetle, Callosobruchus 475 maculatus. Sci Rep. 6:28153.

476 McNamara KB, Sloan NS, Kershaw SE, van Lieshout E, Simmons LW. 2020. Males evolve to be 477 more harmful under increased sexual conflict intensity in a seed beetle. Behav Ecol. doi:10.1093/beheco/arz186. 
479 Michels J, Gorb SN, Reinhardt K. 2015. Reduction of female copulatory damage by resilin represents

480 evidence for tolerance in sexual conflict. J R Soc Interface. 12(104):20141107.

481 doi:10.1098/rsif.2014.1107.

482 Morrow EH, Arnqvist G, Pitnick S. 2003. Adaptation versus pleiotropy: Why do males harm their 483 mates? Behav Ecol. 14(6):802-806. doi:10.1093/beheco/arg073.

484 Le Page S, Sepil I, Flintham E, Pizzari T, Carazo P, Wigby S. 2017. Male relatedness and familiarity 485 are required to modulate male-induced harm to females in Drosophila. Proc R Soc B Biol Sci. 284(1860):11-14. doi:10.1098/rspb.2017.0441.

Parker GA. 2006. Sexual conflict over mating and fertilization: An overview. Philos Trans R Soc B Biol Sci. 361(1466):235-259. doi:10.1098/rstb.2005.1785.

Perry JC, Rowe L. 2012. Sexual conflict and antagonistic coevolution across water strider populations. Evolution (N Y). 66(2):544-557. doi:10.1111/j.1558-5646.2011.01464.x.

Pilastro A, Scaggiante M, Rasotto MB. 2002. Individual adjustment of sperm expenditure accords with sperm competition theory. Proc Natl Acad Sci. 99(15):9913 LP - 9915. doi:10.1073/pnas.152133499.

494 R Development Core Team. 2016. R: A language and environment for statistical computing. R Found 495 Stat Comput. doi:10.1017/CBO9781107415324.004.

496 Rankin DJ, Dieckmann U, Kokko H. 2011. Sexual Conflict and the Tragedy of the Commons. Am 497 Nat. 177(6):780-791. doi:10.1086/659947.

Rice WR. 1996. Sexually antagonistic male adaptation triggered by experimental arrest of female evolution. Nature. 381:232.

500 Ronn J, Katvala M, Arnqvist G. 2007. Coevolution between harmful male genitalia and female 501 resistance in seed beetles. Proc Natl Acad Sci. 104(26):10921-10925. doi:10.1073/pnas.0701170104. 
during mating? Anim Behav. 83(1):75-79. doi:10.1016/j.anbehav.2011.10.007.

504 Rönn JL, Katvala M, Arnqvist G. 2006. The costs of mating and egg production in Callosobruchus 505 seed beetles. Anim Behav. 72(2):335-342. doi:https://doi.org/10.1016/j.anbehav.2005.10.024.

506 Schindelin J, Arganda-Carreras I, Frise E, Kaynig V, Longair M, Pietzsch T, Preibisch S, Rueden C, 507 Saalfeld S, Schmid B, et al. 2012. Fiji: an open-source platform for biological-image analysis. Nat 508 Methods. 9(7):676-682. doi:10.1038/nmeth.2019.

509 Schneider CA, Rasband WS, Eliceiri KW. 2012. NIH Image to ImageJ: 25 years of image analysis. 510 Nat Methods. doi:10.1038/nmeth.2089.

511 Simmons LW, Denholm A, Jackson C, Levy E, Madon E. 2007. Male crickets adjust ejaculate quality 512 with both risk and intensity of sperm competition. Biol Lett. 3(5):520-522.

513 doi:10.1098/rsbl.2007.0328.

514 Sloan NS, Simmons LW. 2019. The evolution of female genitalia. J Evol Biol. 32(9):882-899. 515 doi:10.1111/jeb.13503.

516 Stockley P. 1997. Sexual conflict resulting from adaptations to sperm competition. Trends Ecol Evol. 12(4):154-159. doi:https://doi.org/10.1016/S0169-5347(97)01000-8.

Stoffel MA, Nakagawa S, Schielzeth H. 2017. rptR: repeatability estimation and variance decomposition by generalized linear mixed-effects models. Methods Ecol Evol. 8(11):1639-1644. doi:10.1111/2041-210X.12797.

521 Tatarnic NJ, Cassis G. 2010. Sexual coevolution in the traumatically inseminating plant bug genus 522 Coridromius. J Evol Biol. 23(6):1321-1326. doi:10.1111/j.1420-9101.2010.01991.x.

523 Tatarnic NJ, Cassis G, Siva-Jothy MT. 2014. Traumatic Insemination in Terrestrial Arthropods. Annu 524 Rev Entomol. 59(1):245-261. doi:10.1146/annurev-ento-011613-162111.

525 Thanthianga C, Mitchell R. 1987. Vibrations mediate prudent resource exploitation by competing 526 larvae of the bruchid bean weevil Callosobruchus maculatus. Entomol Exp Appl. 44(1):15-21. 
doi:10.1111/j.1570-7458.1987.tb02233.x.

528 Thomas ML, Simmons LW. 2007. Male Crickets Adjust the Viability of Their Sperm in Response to 529 Female Mating Status. Am Nat. 170(2):190-195. doi:10.1086/519404.

530 Utida S. 1972. Density dependent polymorphism in the adult of Callosobruchus maculatus

531 (Coleoptera, Bruchidae). J Stored Prod Res. 8(2):111-125. doi:https://doi.org/10.1016/0022-

$532 \quad 474 X(72) 90028-8$.

533 Wilson CJ, Buzatto BA, Robinson SP, Tomkins JL. 2014. Sociosexual environment influences

534 patterns of ejaculate transfer and female kicking in Callosobruchus maculatus. Anim Behav. 94:37-

535 43. doi:https://doi.org/10.1016/j.anbehav.2014.05.014.

536 Wilson CJ, Tomkins JL. 2014. Countering counteradaptations: males hijack control of female kicking 537 behavior. Behav Ecol. 25(3):470-476. doi:10.1093/beheco/aru022.

538 Wilson CJ, Tomkins JL. 2015. Female Callosobruchus maculatus can maximize long-term fitness 539 through polyandry. Behav Ecol. 26(2):502-509. doi:10.1093/beheco/aru218.

540 Yamane T, Goenaga J, Rönn JL, Arnqvist G. 2015. Male seminal fluid substances affect sperm

541 competition success and female reproductive behavior in a seed beetle. PLoS One. 10(4):1-14.

542 doi:10.1371/journal.pone.0123770.

543 Zuk M, García-González F, Herberstein ME, Simmons LW. 2014. Model systems, taxonomic bias, 544 and sexual selection: Beyond drosophila. Annu Rev Entomol. 59:321-338. doi:10.1146/annurev-ento545 011613-162014. 
547 Table 1 Fit and loadings for the first two principal components explaining variation in female 548 reproductive tract thickness.

\begin{tabular}{lcc}
\cline { 2 - 3 } & PC1 & PC2 \\
\hline Eigenvalue & $\mathbf{2 . 8 8}$ & 0.78 \\
\% Variance & 71.91 & 19.91 \\
& & \\
Upper mean & 0.930 & 0.25 \\
Upper maximum & 0.777 & 0.60 \\
Lower mean & 0.877 & -0.36 \\
Lower maximum & 0.811 & -0.47 \\
\hline
\end{tabular}

549

550

551 Table 2 Analysis of variance for fixed and random effects on a multivariate measure of

552 female reproductive tract thickness (PC1) (N=54).

\begin{tabular}{lcccc}
\hline & F & df & p & Variance \\
\hline Evolutionary History & 0.51 & $1,3.79$ & 0.52 & \\
Social Environment (SE) & 1.18 & $1,4.38$ & 0.34 & \\
Body Weight & 3.95 & $1,3.61$ & 0.12 & \\
Population replicate & & & & 4.31 \\
Source voltage & & & & $<0.01$ \\
\hline
\end{tabular}

553

554

555

556

557

558

559

560

561 
562 Table 3 Estimated marginal means and effect sizes (Pearson’s r) with 95\% confidence

563 intervals of all fixed effects on a multivariate measure of female reproductive tract thickness 564 (PC1) $(\mathrm{N}=54)$.

\begin{tabular}{ccccc}
\hline & Mean & $\mathbf{9 5 \%}$ CI & $\begin{array}{c}\text { Effect size } \\
\text { (r) }\end{array}$ & 95\% CI \\
\hline $\begin{array}{l}\text { Evolutionary History }(M-F) \\
\quad \text { Female-bias }\end{array}$ & 0.638 & $0.013,1.26$ & -0.28 & $-0.76,0.21$ \\
$\quad$ Male-bias & 0.580 & $-0.17,1.33$ & & \\
Social Environment $(L-H)$ & & & -0.43 & $-0.92,0.05$ \\
$\quad$ High-risk & 0.643 & $0.047,1.24$ & & \\
Low-risk & 0.575 & $-0.15,1.30$ & & \\
\hline
\end{tabular}

565

566 

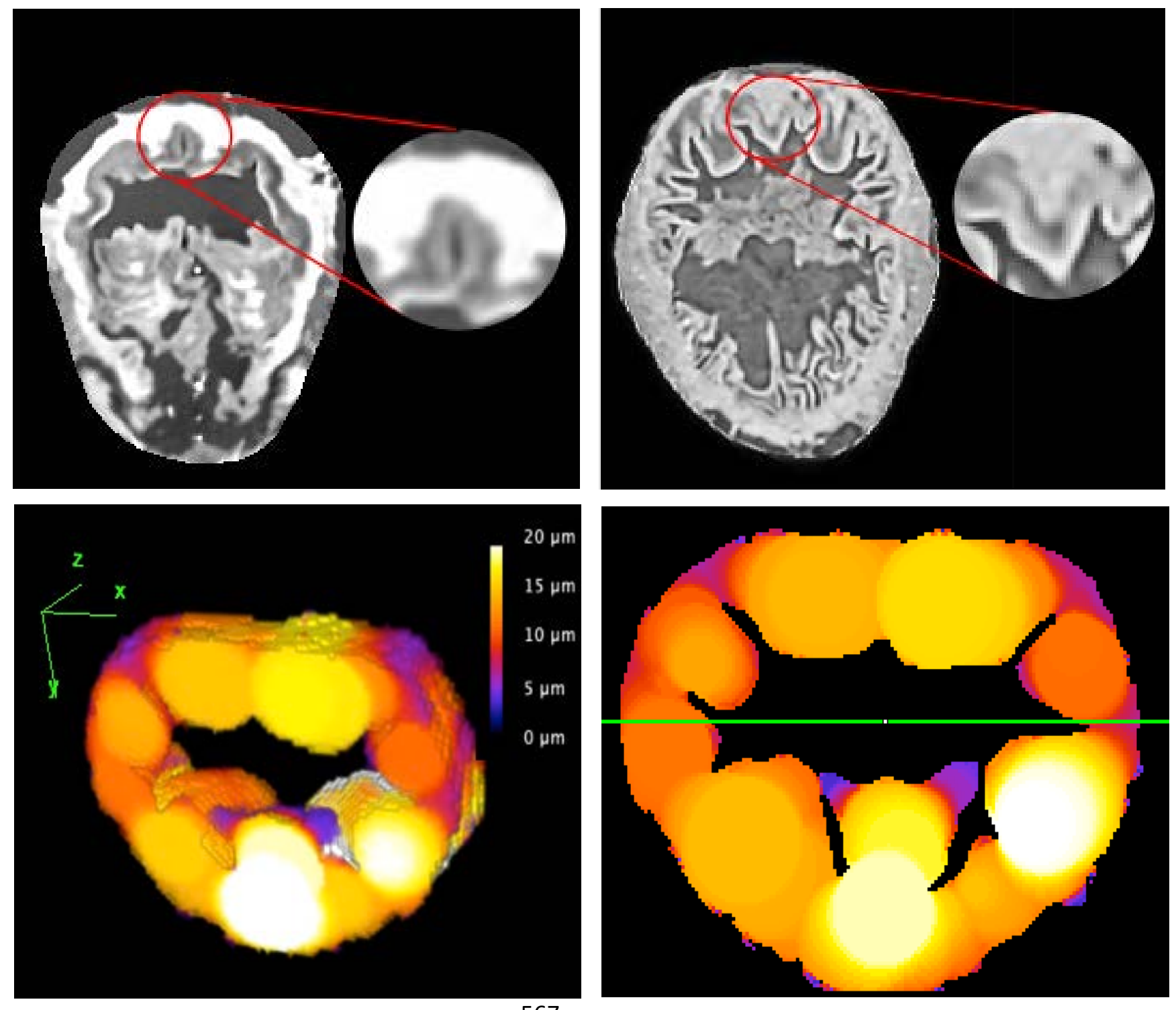

567

568 Figure 1 Segmentation of the reproductive tract: (a) First appearance of the entrance of the

569 spermathecal duct. (b) First appearance of the bursal teeth. Tract wall thickness: (c) 3D

570 thickness heat map (d) Upper and lower regions of interest within the reproductive tract. 